\title{
Neural stem cell sparing by linac based intensity modulated stereotactic radiotherapy in intracranial tumors
}

\author{
Julia Oehler ${ }^{1}$, Tim Brachwitz ${ }^{2}$, Thomas G Wendt ${ }^{1 *}$, Nico Banz ${ }^{2}$, Mario Walther ${ }^{3}$ and Tilo Wiezorek ${ }^{2}$
}

\begin{abstract}
Background: Neurocognitive decline observed after radiotherapy (RT) for brain tumors in long time survivors is attributed to radiation exposure of the hippocampus and the subventricular zone (SVZ). The potential of sparing capabilities for both structures by optimized intensity modulated stereotactic radiotherapy (IMSRT) is investigated.

Methods: Brain tumors were irradiated by stereotactic 3D conformal RT or IMSRT using m3 collimator optimized for PTV and for sparing of the conventional OARs (lens, retina, optic nerve, chiasm, cochlea, brain stem and the medulla oblongata). Retrospectively both hippocampi and SVZ were added to the list of OAR and their dose volume histograms were compared to those from two newly generated IMSRT plans using 7 or 14 beamlets (IMSRT-7, IMSRT-14) dedicated for optimized additional sparing of these structures. Conventional OAR constraints were kept constant. Impact of plan complexity and planning target volume (PTV) topography on sparing of both hippocampi and SVZ, conformity index $(\mathrm{Cl})$, the homogeneity index $(\mathrm{HI})$ and quality of coverage $(\mathrm{QoC})$ were analyzed. Limits of agreement were used to compare sparing of stem cell niches with either IMSRT-7 or IMSRT-14. The influence of treatment technique related to the topography ratio between PTV and OARs, realized in group A-D, was assessed by a mixed model.
\end{abstract}

Results: In 47 patients $\mathrm{Cl}(p \leq 0.003)$ and HI ( $p<0.001)$ improved by IMSRT-7, IMSRT-14, QoC remained stable ( $p \geq 0.50)$ indicating no compromise in radiotherapy. $90 \%$ of normal brain was exposed to a significantly higher dose using IMSRT. IMSRT-7 plans resulted in significantly lower biologically effective doses at all four neural stem cell structures, while contralateral neural stem cells are better spared compared to ipsilateral. A further increase of the number of beamlets (IMSRT-14) did not improve sparing significantly, so IMSRT-7 and IMSRT-14 can be used interchangeable. Patients with tumors contacting neither the subventricular zone nor the cortex benefit most from IMSRT $(p<0.001)$.

Conclusion: The feasibility of neural stem cell niches sparing with sophisticated linac based inverse IMSRT with 7 beamlets in an unselected cohort of intracranial tumors in relation to topographic situation has been demonstrated. Clinical relevance testing neurotoxicity remains to be demonstrated.

Keywords: Stereotactic radiotherapy, Intensity modulated radiotherapy, Brain tumor, Intracranial tumor, Neurocognitive decline, Hippocampus, Subventricular zone, Neural stem cell

\footnotetext{
* Correspondence: Thomas.Wendt@med.uni-jena.de

'Department of Radiation Oncology, Jena University Hospital,

Friedrich-Schiller-University Jena, Bachstrasse 18, Jena D-07743, Germany

Full list of author information is available at the end of the article
} 


\section{Introduction}

Research in the field of neural stem cells has made a quantum leap and yielded complete new insights upon the regeneration of brain cells and functions during the last 15 years. The hippocampal precursor cells, that generate new neurons with their particular function, represent a 'neurogenic reserve' - the potential to remain flexible and plastic in hippocampal learning [1]. Neural stem cells reside in the subventricular zone (SVZ) of the adult mammalian brain. It is concluded that SVZ astrocyte like cells act as neural stem cells in both the normal and regenerating brain [2-4]. The dentate granule cell layer of the hippocampal formation has the distinctive property of ongoing neurogenesis, that continues throughout adult life. Although the function of these newly generated neurons and the mechanisms that control their birth are not yet fully understood. Age, activity and psychosocial stress have all been demonstrated to regulate this type of neurogenesis. Radiation- and chemotherapy-induced damage to progenitor populations, responsible for maintenance of white matter integrity and adult hippocampal neurogenesis, is now believed to play a major role in the neurocognitive impairment many cancer survivors experience $[5,6]$. Therefore functional recovery paved to a major part by cellular restoring may be compromised by radiation therapy.

We explored the potential of modern intensity modulated stereotactic radiotherapy (IMSRT) for sparing the hippocampus, harboring the dentate gyrus and the subventricular zones (SVZ) adjacent to the lateral ventricles.

\section{Material and methods}

All consecutively treated patients, who received high dose fractionated stereotactic radiotherapy for intracranial tumors between $3 / 2007$ and 5/2010, were retrieved from the institutional data base for analysis in this retrospective planning study. Patients with a maximum diameter of the planning target volume (PTV) of $100 \mathrm{~mm}$ and above were excluded. In all patients the tumor to be treated has been confirmed by histology after biopsy, partial or gross tumor resection. The histologic type of tumor is given in Table 1. Anaplastic glioma, glioblastoma (GBM) and gliosarcoma have been conventionally fractionated with 60 Gy /2 Gy (total dose/dose per fraction), low grade glioma with 54 Gy /2 Gy, all other entities with 50.4 to 54 Gy /1.8 Gy (ICRU 50). Patients with unconventional fractionation schedules were excluded. Patients with GBM and anaplastic glioma WHO ${ }^{\circ} 3$ or ${ }^{\circ} 4$ were given temozolomide, $75 \mathrm{mg} /$ sqm body surface area, orally every treatment day. The technique applied was selected in order to match the conventional dose constraints as good as possible. Clinically applied treatment plans were generated by the following procedures and planning constraints.
Table 1 Demographic and tumor characteristics and radiotherapy planning details

\begin{tabular}{|c|c|}
\hline n (male: female) & $47(26: 21)$ \\
\hline median age (range) & 49.68 years $(2-88)$ \\
\hline \multicolumn{2}{|l|}{ histology } \\
\hline meningioma & 14 \\
\hline anaplastic astrocytoma & 11 \\
\hline glioblastoma & 8 \\
\hline oligodendroglioma & 5 \\
\hline craniopharyngeoma & 4 \\
\hline oligoastrocytoma & 1 \\
\hline xantoastrocytoma & 2 \\
\hline gliosarcoma & 1 \\
\hline rhabdomyoid tumor & 1 \\
\hline 3 D conformal plan ${ }^{a}$ & 27 \\
\hline micromultileaf collimator (m3) IMSRT a & 20 \\
\hline \multicolumn{2}{|l|}{ total radiation dose (ICRU 50) } \\
\hline mean \pm SD (range) & $55.19 \pm 3.77(50-60) \mathrm{Gy}$ \\
\hline \multicolumn{2}{|l|}{ PTV volume } \\
\hline mean \pm SD (range) & $79.13 \pm 66.62(2.1-255.3) \mathrm{ml}$ \\
\hline \multicolumn{2}{|l|}{ topographic groups $^{b}(n)$} \\
\hline A & 11 \\
\hline $\mathrm{B}$ & 8 \\
\hline C & 14 \\
\hline D & 14 \\
\hline
\end{tabular}

: to fulfil of conventionally defined organs at risk constraints (see text)

b. according to the definition of [10].

\section{Conventional planning procedure}

For planning purposes all patients had a plain axial computed tomography $(\mathrm{CT})$ with $2.5 \mathrm{~mm}$ slice thickness, covering the entire brain from the vertex to cervical vertebra 2. The CT data set was taken on a scanner dedicated for radiotherapy planning. The patient was fixed in the high precision mask system, encaged in the stereotactic CT localizer (Brainlab ${ }^{\circ}$, Feldkirchen/Munich, Germany). A 1.5 Tesla magnet resonance image (MRI) rendering volume scan based on 192 sagittal slices with $0.9 \mathrm{~mm}$ thickness was acquired immediately after contrast injection for all patients to enhance contouring. For delineation, a gadolinium-enhanced T1-weighted gradient-echo sequence was used to delineate gross tumor volume (GTV), clinical target volume (CTV) and planning target volume (PTV). $\mathrm{CT}$ and MRI data sets were fused using the integrated fusion algorithm of the planning software iPlan $\mathrm{RT}^{\bullet}$ version 3.0 and 4.0 (Brainlab, Feldkirchen/Munich, Germany).

GTV was contoured according to the contrast enhanced volumes. The CTV was generated by contouring a margin around the surgical defect (tumor bed) accounting for microscopic extension in case of incomplete surgical 
removal or biopsy only. The margin around GTV to create CTV was chosen $10 \mathrm{~mm}$ in high grade tumors (WHO ${ }^{\circ} 3$ and 4) and 5-7 mm unless the scull would have been exposed in low grade tumors ( $\mathrm{WHO}^{\circ} 2$ ). For meningioma WHO grade 1 and craniopharyngioma, the selected margin was $2-3 \mathrm{~mm}$. PTV was generated by an additional margin of $2 \mathrm{~mm}$ in all directions, irrespective of tumor grading to compensate for daily set-up error.

The following structures (conventional organs at risk, OARs) were routinely delineated with their maximum dose accepted in the final plan (constraints given in brackets): lens (10 Gy), retina (35 Gy), optic nerves (45-50 Gy), chiasm (45 Gy), cochlea (45 Gy), brain stem (50 Gy) and medulla oblongata (50 Gy). Contouring was performed in axial reconstructions of the MRI data set. All plans were calculated and optimized in Brainlab iPlan $\mathrm{RT}^{\bullet}$ version 3.0 and 4.0 and applied to the patient using the micromultileaf collimator m3 (Brainlab, Feldkirchen/Munich, Germany). The preferred treatment plan used forward planned 3D conformal non-coplanar isocentric fixed beams. If 3D conformal RT planning failed to meet any of these constraints, IMSRT using the step-and-shoot technology was used. An experienced medical physicist and a radiation oncologist decided on the applied technology on an individual basis. All patients were irradiated with plans optimized for sparing of delineated OARs and yielding acceptable dose homogeneity at the PTV. The ICRU 50 criteria with regard to PTV have been fulfilled in all plans. These plans have been denominated as "not optimized" to describe the results. The normal tissue maximum doses have been recorded from the dose volume histograms (DVH) generated for all three plans in each patient.

\section{Planning optimized for hippocampal and subventricular zone (SVZ) sparing}

Neural stem cell niches were delineated in all restored original MRI or CT data sets (Figure 1) for the prospective optimization process. Left and right hippocampi were contoured with the assistance of an experienced neuroradiologist, according to published guidelines [7]. The left/right SVZ were contoured around left/right lateral and third ventricles, creating a 3-5 $\mathrm{mm}$ rim immediately adjacent to their lateral borders [8]. The total volume of the brain from the vertex down to the foramen magnum was calculated after the PTV has been excluded, using Boolean operators. Thus the mean dose applied to $10 \%$ (D 10), 50\% (D 50) and 90\% (D 90) of the normal brain volume was generated for all planning procedures in all patients.

Two intensity modulated plans were generated and optimized for the additionally contoured hippocampi and SVZ. In order to maintain the constraints of conventionally contoured OARs, the beam directions had been left unchanged. The technique of intensity modulated stereotactic

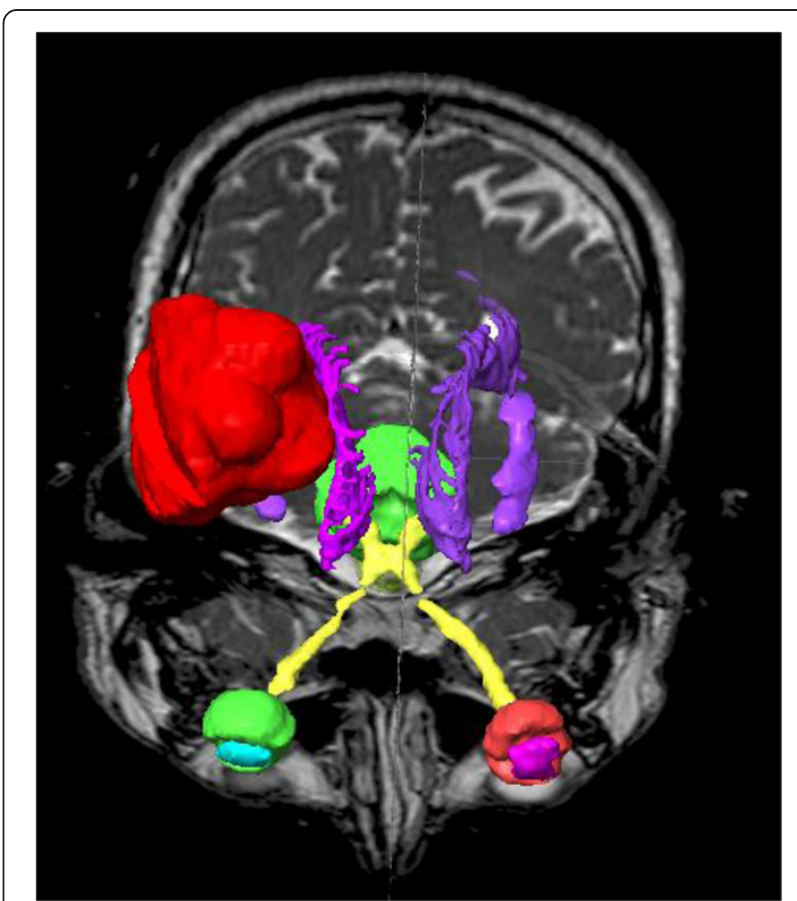

Figure 1 Right temporal planning target volume (PTV, red) and organs at risk delineated in the MRI rendering volume data set. Lenses in pale blue and purple, nn. optici and chiasm in yellow, brain stem and medulla oblongata in green, the hippocampi and the subventricular zones (SVZ) in different shades of magenta.

radiotherapy was already used in daily routine during study runtime. Our experiences resulted in a number of beamlets varying between 10 and 20. 14 beamlets were a satisfying approach for all cases used in this study. It was a goal to reduce the number of beamlets ( 7 beamlets) with the aim to get robust plans. As a positive side effect, these kind of plans provide shorter treatment times. In addition to that a better agreement between measured and calculated isodoses were expected, by avoiding very small beamlets. Therefore the first plan was generated using a leaf sequencer setup of 7 beamlets per beam; the second plan was generated using 14 beamlets per beam in order to optimize photon fluence profiles. For describing the results, these plans have been denominated "IMSRT-7" and "IMSRT-14" respectively. The constraints for the hippocampus and the SVZ were set at 6 Gy maximum total dose each. The maximum doses for hippocampi and SVZ obtained in the new plans were transformed into biologic effective doses (BED) using an alpha-beta ratio of 2 in the linear quadratic model.

The PTV was subject to an analysis of parameters describing the quality of dose distribution. The conformity index (CI) was defined as target volume (TV) divided by the volume receiving the reference dose (VRI) [9]. The homogeneity index (HI) was defined as maximum isodose in the target (Imax) divided by the reference isodose (RI). 
The quality of coverage (QoC) was defined as the minimal isodose encompassing the target (Imin) divided by reference isodose (RI).

To explore the impact of topographic tumor site on quality indices and on potential hippocampus and SVZ sparing using IMSRT-7 and IMSRT-14 techniques, PTVs were assessed for their contact with the hippocampus and/or SVZ and the cortex as described elsewhere [10,11]. Four groups were segregated: group A (tumors contacting the SVZ and infiltrating cortex), group B (tumor contacting SVZ but not involving the cortex), group C (tumor involving cortex but not contacting the SVZ), group D (tumor involving neither SVZ nor cortex) (Figure 2).

\section{Statistics}

The software SPSS (version 18.0; SPSS, Chicago, IL, USA) was used to describe and analyse non-normal distributed data. Normal brain exposure and quality indices of the planning treatment volume, after different planning modes were tested using the non-parametric Wilcoxon-(rank sum) - test.

It was hypothesized that the following variables will impact the dose at the neurogenic stem cell niches: the structure to be spared (both hippocampi and SVZ), the topographic site of PTV (group A-D), its laterality (ipsilataral or contralateral to the PTV) and the mode of planning (not optimized, IMSRT-7, IMSRT-14). The agreement of biologic radiation doses for IMSRT-7 and IMSRT-14 plans at the hippocampi and the SVZ was visualized by Bland-Altman plot [12], which is a method to analyse the agreement of two different techniques by graphical presentation. For each structure-laterality-combination (Hippocampus/SVZ-ipsilateral/contralateral) of the two planning strategies (IMSRT-7; IMSRT-14), the individual differences of BED between IMSRT-14 and IMSRT-7 are plotted against their respective averages. The limits of agreement, which are given by mean value $+/-1.96$ times the standard deviation of the differences, are computed also. These limits of agreement yield an area including 95\% of the differences. Instead of using the original BED-data for the Bland-Altman plot the log-transformed BED-data were used, because a first inspection of the Bland-Altman plot with the original data showed systematic changes in the differences.

For this reason the BED data were also estimated in consideration of structure, group, laterality and treatment plan by applying linear mixed model using SAS 9.3. Thereby it is possible to consider and calculate the individual dependency concerning treatment plan, laterality and analyzed structures. The model for assessing the correlations between the different treatment techniques was based on unstructured covariance patterns. Concerning intraindividual factors of laterality and structure, no correlations are expected in terms of measured BEDs, which are also considered in the calculated model. In all analyses a p-value $<0.05$ was considered as statistically significant. To account for multiplicity we applied Bonferroni adjustment.
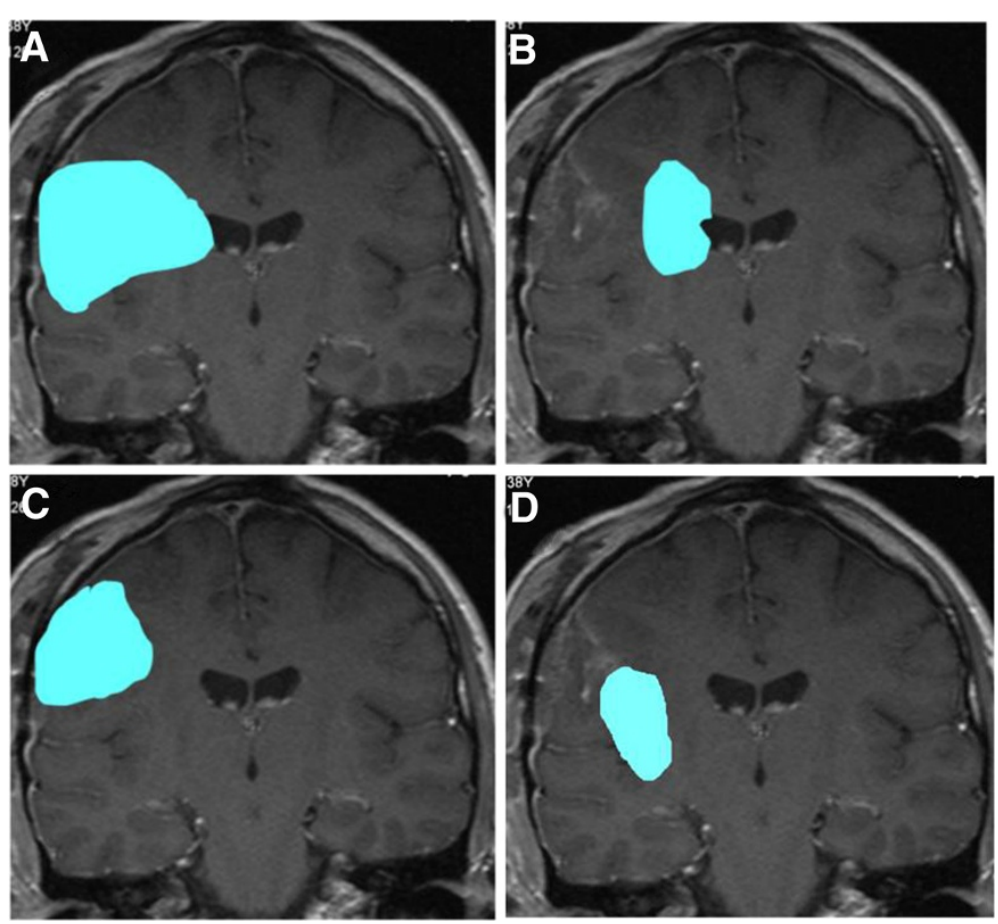

Figure 2 Topographic type of 47 intracranial tumors: panels A -D modified according to [10]. 


\section{Results}

Demographic and tumor characteristics of 47 patients studied are given in Table 1. The tumor was located for 18 patients in the left hemisphere, for 18 patients in the right hemisphere and for 11 patients centrally. In these cases the tumor was arbitrarily allocated to the left or right hemisphere for analysis purposes according to its dominating laterality. To fulfil the conventional constraints, 27 patients received $3 \mathrm{D}$ conformal plans and 20 patients initially m3-IMSRT in their original treatment plans without optimizing for hippocampi and SVZ. Dose constraints were respected in all conventionally contoured organs at risk in all plans. For optimized IMSRT-7 and IMSRT-14 plans the maximum doses for these OARs remained unchanged, while an optimization was performed for sparing of both hippocampi and SVZ. Topographic groups A, B, $\mathrm{C}$ and $\mathrm{D}$ comprised in $11,8,14$ and 14 patients respectively (Table 1, Figure 2). None had multifocal tumor.

Normal brain exposure, conformity index, homogeneity index and quality of coverage

The dose administered to $10 \%, 50 \%$ and $90 \%$ of the normal brain volume, outside the target volume (D 10, D 50, and D 90) varies for each treatment modality and proofed independent from the planning procedure (Table 2). However, when $90 \%$ of the normal brain volume is considered optimized, planning with IMSRT-7 or IMSRT-14 results in a small but significantly higher radiation exposure compared to the not optimized plans. D 90 slightly further increased using 14 beamlets compared to 7 (Table 2). Indeed exposure of a larger normal tissue volume at low dose levels is a hallmark of increasing number of beamlets in IMSRT. Both CI and HI of planning treatment volume show a small but significant improvement for the optimized IMSRT-7 and to the IMSRT-14 plans compared to the not optimized plans, albeit plans were not optimized for these parameters. The quality of coverage of the IMSRT plans compared to the not optimized plans remained unchanged (Table 2). Overall hippocampus and SVZ sparing was not achieved at the expense of quality of dose distribution at the target volume.

Itemized observation of parameters (laterality, plan, group, structure)

For the entire group $(n=47)$ dose reduction was achieved by IMSRT 7 and IMSRT 14. Group D had noticeable lower

Table 2 Normal brain radiation exposure (median doses [Gy] and 25\%- and 75\%-quartiles) and conformity index, homogeneity index and quality of coverage of the planning target volume

\begin{tabular}{|c|c|c|c|c|c|c|}
\hline & $\begin{array}{l}\text { Not optimized } \\
\text { plan a }\end{array}$ & $\begin{array}{l}\text { Optimized } \\
\text { IMSRT-7 }\end{array}$ & $\begin{array}{l}\text { Optimized } \\
\text { IMSRT-14 }\end{array}$ & $\begin{array}{l}\text { Not optimized vs. } \\
\text { IMSRT-7 p }\end{array}$ & $\begin{array}{l}\text { Not optimized vs. } \\
\text { IMSRT-14 p }\end{array}$ & $\begin{array}{l}\text { IMSRT } 7 \text { vs. } \\
\text { IMSRT-14 p }\end{array}$ \\
\hline \multicolumn{7}{|c|}{ Whole brain exposure } \\
\hline \multirow[t]{3}{*}{ D $10(G y)^{b}$} & 25.290 & 26.240 & 24.450 & 0.563 & 0.443 & 0.783 \\
\hline & Q25: 13.090 & Q25:12.280 & Q25: 12.400 & & & \\
\hline & Q75: 33.820 & Q75: 34.740 & Q75: 33.300 & & & \\
\hline \multirow[t]{3}{*}{ D 50 (Gy) b } & 7.000 & 6.510 & 6.500 & 0.589 & 0.663 & 0.829 \\
\hline & Q25: 3.960 & Q25: 3.550 & Q25: 3.480 & & & \\
\hline & Q75: 10.900 & Q75: 10.080 & Q75: 10.100 & & & \\
\hline \multirow[t]{3}{*}{ D 90 (Gy) b } & 1.400 & 1.900 & 1.950 & $<0.001$ & $<0.001$ & 0.423 \\
\hline & Q25: 1.100 & Q25: 1.420 & Q25: 1.460 & & & \\
\hline & Q75: 3.280 & Q75: 3.300 & Q75: 3.280 & & & \\
\hline \multirow{3}{*}{$\begin{array}{l}\text { conformality } \\
\text { index }{ }^{c}\end{array}$} & 0.980 & 0.990 & 1.000 & 0.003 & $<0.001$ & 0.005 \\
\hline & Q25: 0.970 & Q25: 0.990 & Q25: 0.990 & & & \\
\hline & Q75: 1.000 & Q75: 1.000 & Q75: 1.000 & & & \\
\hline \multirow{3}{*}{$\begin{array}{l}\text { homogeneity } \\
\text { index }{ }^{c}\end{array}$} & 1.140 & 1.110 & 1.090 & $<0.001$ & $<0.001$ & $<0.001$ \\
\hline & Q25: 1.100 & Q25: 1.090 & Q25: 1.070 & & & \\
\hline & Q75: 1.170 & Q75: 1.120 & Q75: 1.110 & & & \\
\hline \multirow{3}{*}{$\begin{array}{l}\text { quality of } \\
\text { coverage }^{c}\end{array}$} & 0.920 & 0.930 & 0.920 & 0.560 & 0.500 & 0.757 \\
\hline & Q25: 0.850 & Q25: 0.890 & Q25: 0.880 & & & \\
\hline & Q75: 0.950 & Q75: 0.930 & Q75: 0.940 & & & \\
\hline
\end{tabular}

Comparison between not optimized dose distribution and plans optimized for hippocampus and SVZ sparing by intensity modulated stereotactic radiotherapy using 7 or 14 beamlets (IMSRT-7, IMSRT-14). p: p-value from non-parametric Wilcoxon-(rank sum)- test.

${ }^{a}$ : 27 plans: $3 \mathrm{D}$ conformal, 20 plans: IMSRT optimized for conventionally contoured organs at risk.

b : D10, D50, D90: the median dose (Gy) + quartile to 10, 50 and $90 \%$ of the normal brain volume (ml) left after the PTV volume was excluded.

c : conformity index: VRT/TV, homogeneity index: Imax/R, quality of coverage: Imin/RI. 
BED doses compared with groups A-C overall. Achieved levels of sparing were favorable when the PTV was located contralateral to hippocampus and SVZ (Figure 3 A-D).

In line with the linear mixed model significances regarding variables structure to be spared (both hippocampi and SVZ), the topographic site of PTV (group A-D), its laterality (ipsilataral or contralateral to the PTV) and the mode of planning (not optimized, IMSRT-7, IMSRT-14) were observable $(\mathrm{p}<0.0001)$, which could not be interpreted independent due to interactions (Table 3). No significances can be proved between laterality and structures as well as between laterality or structures and different treatment plans. In contrast laterality, structures and treatment plans interact with group classification significantly $(\mathrm{p}<0.0001 /$ $\mathrm{p}=0.0013 / \mathrm{p}=0.0062$ ). Due to inconclusiveness no additional higher-order interdependency could be established ( $p>0.1237$ ). A significant difference exists for the laterality concerning group $\mathrm{A}, \mathrm{B}$ and $\mathrm{C}(\mathrm{p}<0.0001)$ and for the structures regarding to group $\mathrm{A}$ and $\mathrm{B}(\mathrm{p}<0.0001 ; \mathrm{p}=0.0028)$ on a closer examination of group assignment. Referring to treatment plans significant differences for group B, C and D are verifiable $(\mathrm{p}<0.0001)$, but even more precisely for IMSRT 7 and IMSRT 14 in comparison with the not optimized treatment plans. Between IMSRT 7 and IMSRT 14 techniques no statistically significant difference is detected in each group.

Group assignment has an effect on laterality, structures and treatment plans, whereas the interaction between treatment plans and laterality, respectively structure is not significant. For group B, C and D is it possible to optimize the treatment plan concerning organs at risk with IMSRT 7 as well as IMSRT 14 compared to not optimized plans.

Comparison of 7 and 14 beamlets IMSRT concerning OARs

A Bland-Altman plot displays the agreement between der IMSRT-7 and IMRST-14 technique including the limits of agreement for each organ at risk (Figures 4, 5, 6, 7). A slight bias about the mean for ipsi- and contralateral hippocampus and SZV with marginal numbers of outliers beyond limits of agreement can be observed. The 14 beamlets IMSRT techniques provides total doses to contralateral hippocampus and SVZ, which ranges from 15\% and 19\% smaller, till 13\% and $25 \%$ higher than with the 7 beamlets technique, respectively. The ipsilateral total doses for hippocampus and SVZ ranges from $93 \%$ to $108 \%$ and $87 \%$ to $116 \%$ with IMSRT-14 compared to IMRST-7. The total doses for the ipsilateral hippocampus is in 95\% of all IMSRT-14 plans not more than $8 \%$ higher or $7 \%$ lower compared to IMSRT-7 plans.

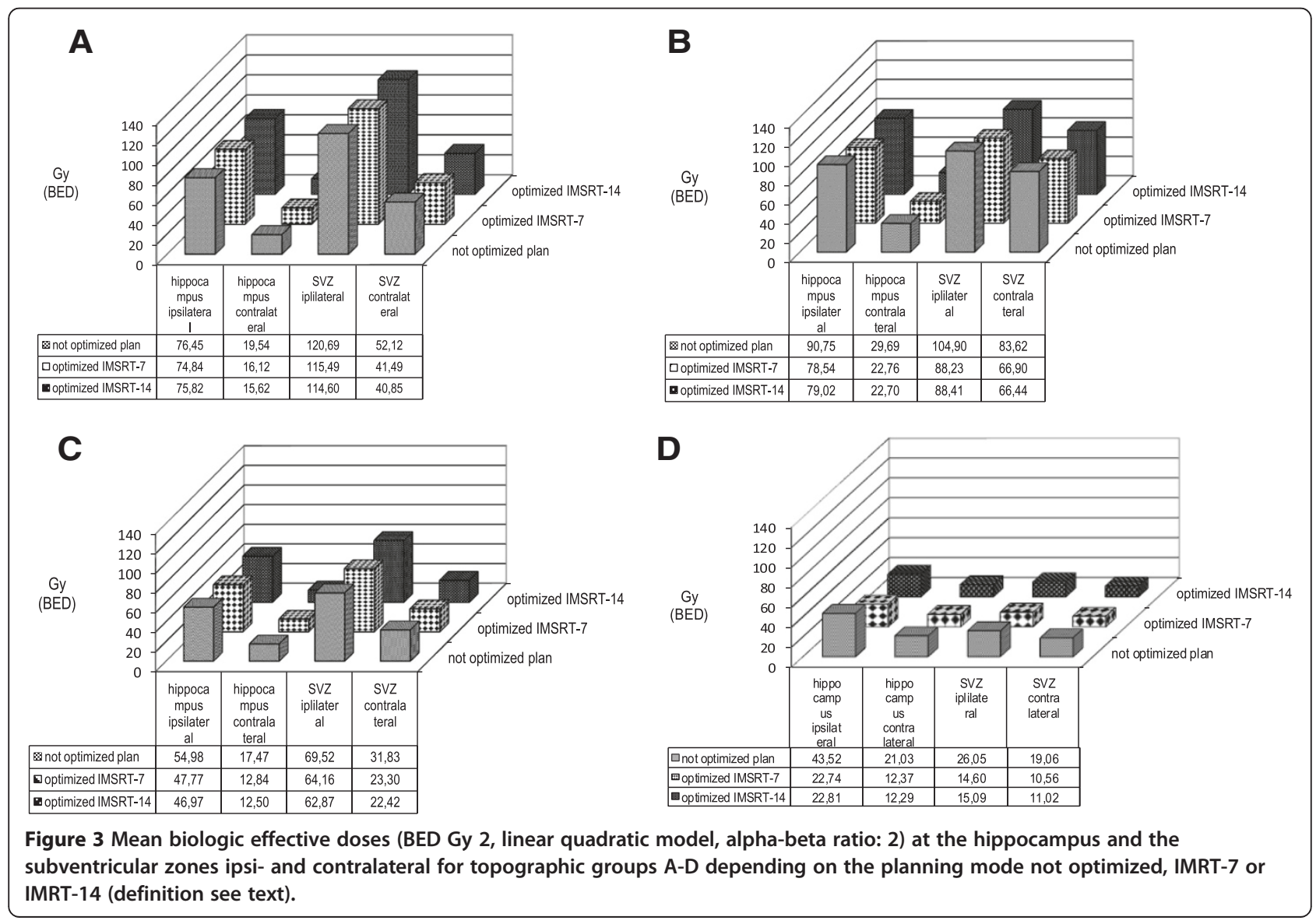


Table 3 Tests of constant effects, $p \leq \mathbf{0 . 0 0 5}$

\begin{tabular}{ll}
\hline Effect & p-value \\
\hline laterality & $<0.001$ \\
structure & $<0.001$ \\
laterality* structure & 0.39 \\
plan & $<0.001$ \\
laterality * plan & 0.62 \\
structure*plan & 0.24 \\
group & $<0.001$ \\
laterality*group & $<0.001$ \\
structure*group & 0.00 \\
plan*group & 0.01 \\
\hline
\end{tabular}

* is an indication for multi structure analysis ( 2 together).

For the ipsilateral SVZ, 95\% of all total dose figures for the IMSRT-14/IMSRT-7 ratio are within the range of $87 \%$ to $116 \%$, i.e. it is expected that IMSRT-14 dose is not more than $13 \%$ lower or $16 \%$ higher compared to the IMSRT-7 dose. Furthermore, a small bias is inherent, when doses at contralateral hippocampus and SVZ are calculated using IMSRT-14. Techniques can be used interchangeable.

Adding them to the inverse optimization target function of a dedicated IMSRT considerably reduces the dose burdens at hippocampi and SVZ. Sparing at these structures critically depends on the relationship between the site of the tumor and the organ to be spared and its laterality. It seems reasonable to assume, that localization of tumor related to SVZ and hippocampus has an important impact on choice of treatment technique.

\section{Discussion}

Neurotoxicity after radiotherapy has been classified as acute, subacute and late. Beside a variety of morphological changes detected in MRT (e.g. white matter lesions) and histologically, neurocognitive changes observed particularly in long term survivors have been associated with brain irradiation [6]. It is assumed, that the clinical counterpart of diffuse white matter changes may be a declined neurocognitive function, observed in children after fractionated total doses as low as 18 Gy to 24 Gy in 2 Gy fractions (BED $=36 \ldots$ $48 \mathrm{~Gy}_{2}$ using $\alpha / \beta$ ratio of 2 ) [13]. This issue is of growing importance since survival has risen gradually over the past decade in virtually all malignant brain tumors. Recent reports on anaplastic glioma and GBM demonstrated an increased survival approaching a median of two years after escalated doses, e.g. applied by a stereotactic boost or by re-irradiation after recurrence, but also adverse long term neurotoxicity [14-17]. However, with more sensitive testing tools, neurocognitive decline can be detected as early as 4 months. After whole brain irradiation for brain metastases with $30 \mathrm{~Gy}, 52 \%$ of patients show a decline in learning and memory function compared to $24 \%$ in patients treated with stereotactic irradiation for 1-3 brain metastases alone [18]. Toxicity data from low-grade glioma patients treated with radiation doses of 50 Gy reported an actuarial 5-year incidence of mental decline of 5.3\% [19]. Neurocognitive changes may be detected after a long clinically silent period up to 12 years after radiotherapy for anaplastic oligodendroglioma brain tumours [20], albeit cognitive dysfunction may be present before treatment begun [21]. Several factors are known to impact the development of

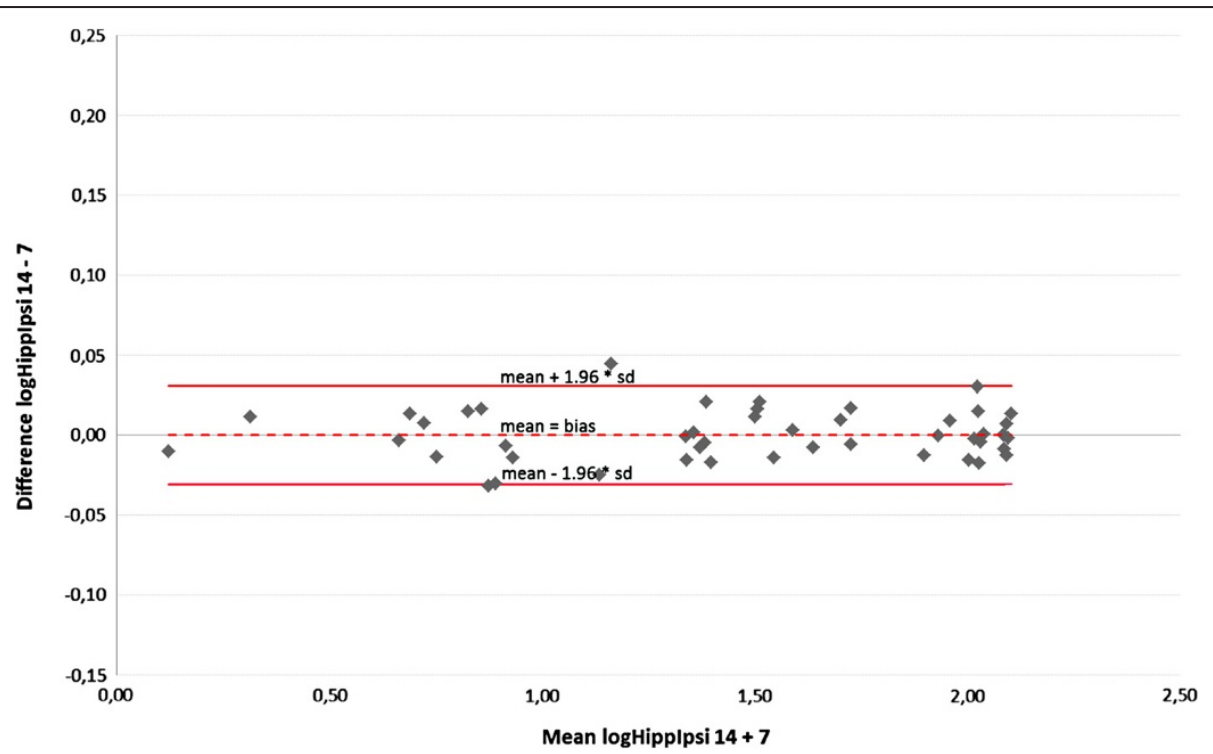

Figure 4 Mean and standard deviation of differences between total doses at the hippocampus ipsilateral to the PTV when irradiated with IMSRT-14 vs. IMSRT-7 in 46 patients (Bland-Altman plot); limits of agreement (mean+/-1.96*SD) in original scale: $+8 \% /-7 \%$. 


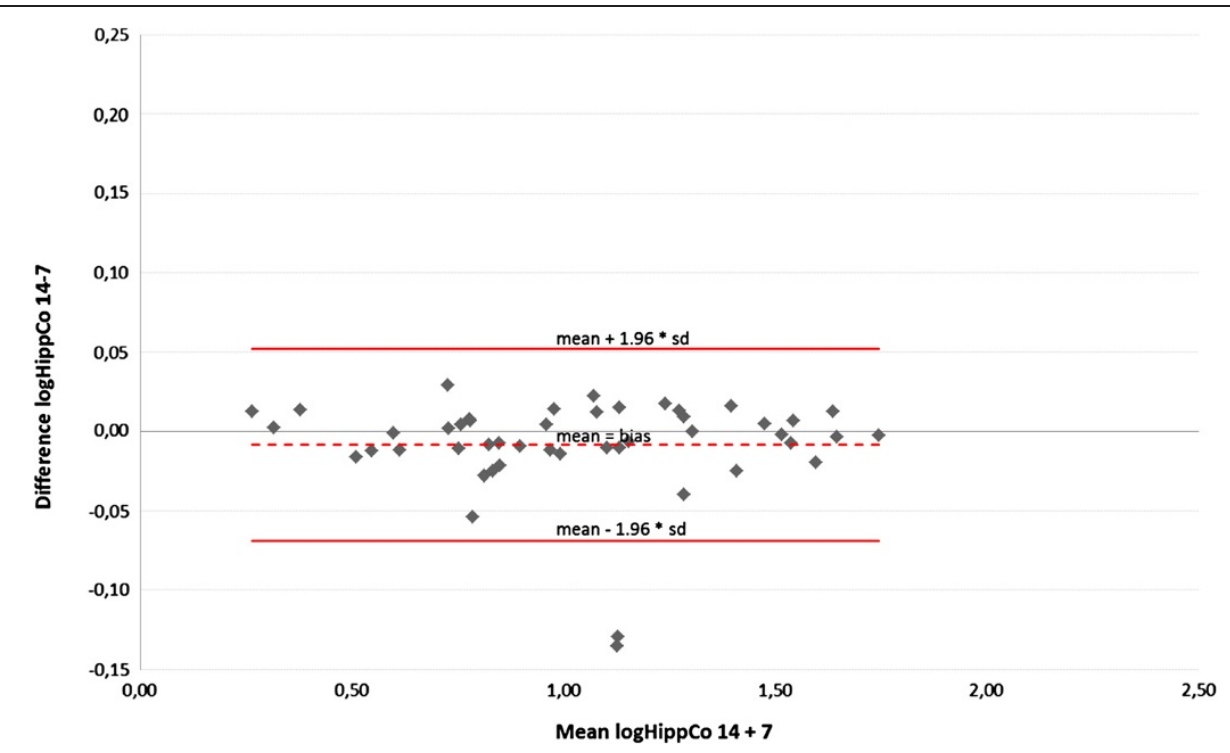

Figure 5 Mean and standard deviation of differences between total doses at the hippocampus contralateral to the PTV when irradiated with IMSRT-14 vs. IMSRT-7 in 47 patients (Bland-Altman plot); limits of agreement (mean+/-1.96*SD) in original scale: $+13 \% /-15 \%$.

neurocognitive disturbances; however a correlation between radiation dose and risk or severity of findings has not been established.

Extensive experimental research during the last 15 years has begun to elucidate the pathophysiologic mechanisms that may underlie neurocognitive dysfunction after radiotherapy of the brain. Radiotherapy and chemotherapy impact on normal (adult) neurogenesis originating in brain stem cells localized in dentate gyrus of the hippocampus and the subventricular zones around the lateral ventricles $[6,22,23]$.

Hippocampal neurogenesis is a very complex physiological process [1]. Neuronal stem cells localized in the subventricular zone are considered to maintain white

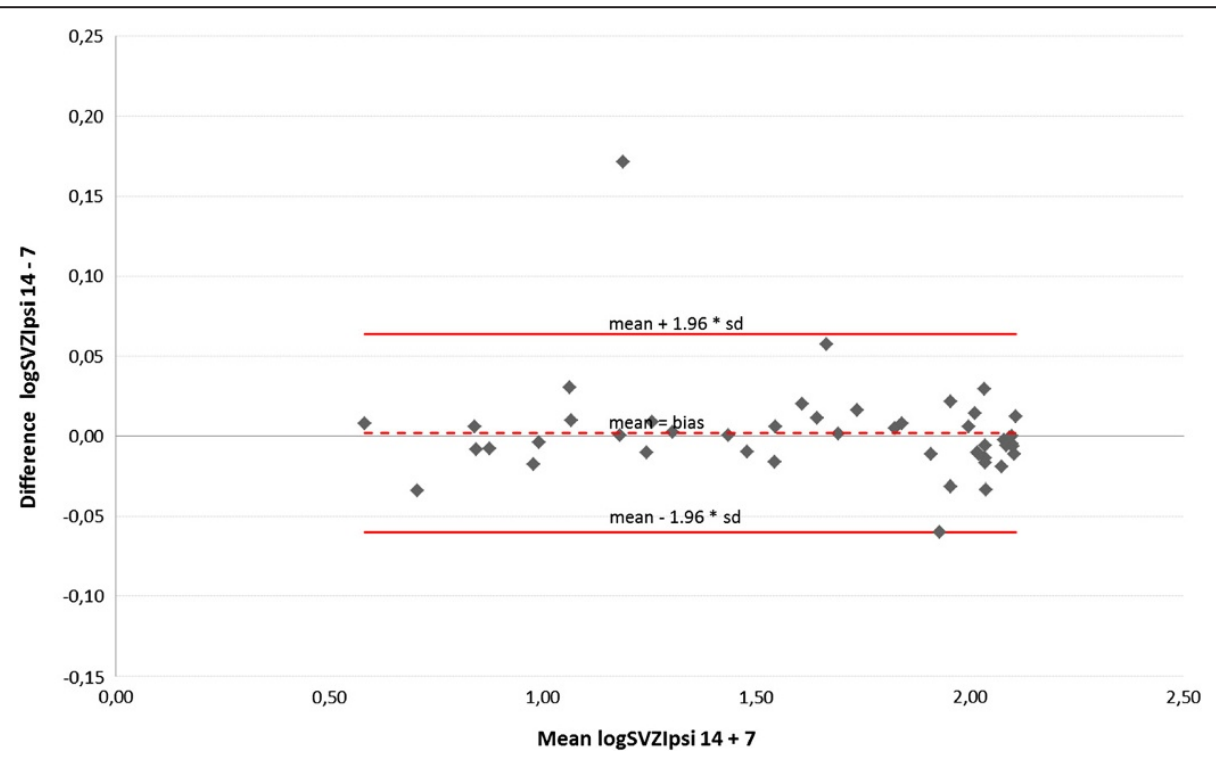

Figure 6 Mean and standard deviation of differences between total doses at the subventricular zone ipsilateral to the PTV when irradiated with IMSRT-14 vs. IMSRT-7 in 47 patients (Bland-Altman plot); limits of agreement (mean+/-1.96*SD) in original scale: $+16 \% /-13 \%$. 


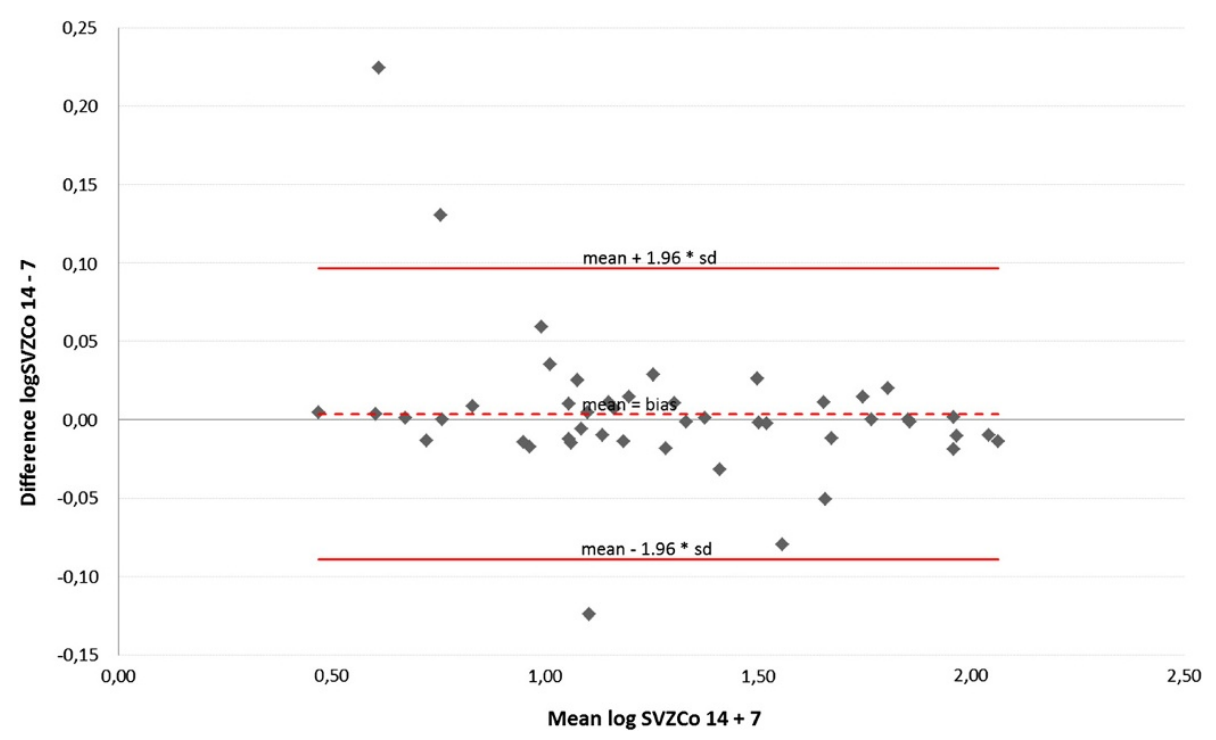

Figure 7 Mean and standard deviation of differences between total doses at the subventricular zone contralateral to the PTV when irradiated with IMSRT-14 vs. IMSRT-7 in 47 patients (Bland-Altman plot); limits of agreement (mean+/-1.96*SD) in original scale: $+25 \% /-19 \%$.

matter integrity. Generation of new neurons in the dentate gyrus of the hippocampus occurs in both rats and humans throughout life, and the addition of these new neurons is believed to be important to normal memory function [24-26]. Experimental and human studies have documented that radiation is able to disrupt the neurogenesis in the adult dentate gyrus even after exposure to single doses as low as 6 Gy, which is equivalent to 12 Gy given in 6 fractions with 2 Gy each (both resulting in BED of $24 \mathrm{~Gy}_{2}$ assuming a $\alpha / \beta$ ratio of 2$)[4,23,27-30]$. This may contribute to the clinical finding of neurocognitive decline after radiotherapy performed with low doses for brain metastases. Due to the proximity of the target volumes and their exposition with high doses while simultaneous sparing of hippocampal structures, the constraints for the latter may only be accomplished in a subgroup of tumor and / or using sophisticated and dedicated radiotherapy planning procedures. The present series demonstrate better sparing of contralateral neural stem cell niches compared to ipsilateral. The topographic site of PTV seems to impact the degree of sparing capability. In the present series, total doses at the neural stem cell niches are below doses associated with neurogenic impairment (after whole brain irradiation in children and adults) and even the dose range leading to a disruption of the neurogenesis in mouse models particularly in patients of group D (tumor involving neither subventricular zone nor cortex). Patients with such tumors may benefit most from dedicated IMSRT planning.

In conventional radiotherapy planning however neither the hippocampus nor the SVZ are contoured and considered as organs at risk during the topographical dose optimization process. Better sparing of normal brain tissue has been accomplished with IMSRT substituting 3D conformal dose escalated radiotherapy for high grade glioma with a consecutively reduced normal tissue complication probability in a radiobiological modelling study [31]. In patients requiring whole brain irradiation due to brain metastases, IMSRT has been shown to better spare the hippocampus both by linac based technique as well as by tomotherapy, when these organs at risk have been subjected to a high penalty in the inverse optimization algorithm $[14,32,33]$.

IMSRT-7 and IMSRT-14 was optimized for sparing of the hippocampi and the SVZ. The dose homogeneity at the PTV as well as conformity index improved as an unscheduled side effect. The data presented demonstrate a considerable sparing achieved by contouring neuronal stem cell niches and exploiting the capabilities of micromultileaf collimation and dedicated dose optimization in a stereotactic treatment setting. The extent of sparing depends on the size of PTV but also its topographic site described by its relation to the cortex and the lateral ventricles. Additional modelling of high isodoses had only a marginal impact on sparing. This may be a valuable finding because it has the potential to prevent physicists from additional planning burden.

The Bland-Altman plot is a graphical method to show the relation between the differences and averages of two techniques, to control for systematic bias and to identify outliers. For clinically evaluation and interpretation of the data a back-transform of logarithm is practicable. The clinical importance of advancement between the two techniques can be measured against each other on the basis of percentages in original scale. The Bland-Altman plot also 
enables a statement relating to the comparison of IMSRT with 7 and 14 beamlets. Thereby no advantages with 14 beamlets over 7 beamlets are detectable.

The analysis seems reasonable to assume that localization of tumor related to SVZ and hippocampus has an important impact on choice of treatment technique. It is possible to optimize the treatment plan concerning organs at risk with IMSRT-7 as well as IMSRT-14 compared to non optimized plans for group B, C and D. On the contrary the different treatment techniques have no preserving effect for organs at risk when the tumor contacts SVZ and cortex irrespective of laterality.

The patient bases of this study were cases already treated with 3D-conformal SRT. The optimal beam directions for the 3D-SRT plans avoid direct interaction with any OAR and were already optimized by experienced medical physicists. The beam directions have been left unchanged because they allowed best sparing and were the ideal solution for every case. It is in our opinion highly recommended to use non-coplanar beam setups for cranial indications due to increase the number of degrees of freedom.

The aim of modern planning is not only to reduce the doses at the remaining normal brain and standard OAR, which has been correlated with different treatment methods [34], but also to spare sites where neurogenesis takes place, more specifically. However, an unsolved issue is the dose to be targeted at the hippocampus and the SVZ particularly according to the obviously multifactorial impairment of stem cell regeneration, in which corticoids and anticonvulsive drugs interfere with radiation [5]. In this series a total dose of 6 Gy over the entire series of fractionated radiotherapy was used as dose constraint. Others set the dose to zero, which seems unrealistic [8]. Recently a dose of 7 Gy at the hippocampus has been shown to impair late memory [35]. This figure could arbitrarily serve as a constraint in IMSRT however needs further confirmation.

\section{Competing interests}

Thomas G. Wendt, Tilo Wiezorek and Nico Banz received honorarium from Brainlab AG independent from this work. All other authors declare that they have no competing interests.

\section{Authors' contributions}

JO carried out the contouring of critical structures, data management, performed the data analysis and statistics, reviewed the literature and contributed to writing the manuscript. TB prepared data set for contouring and generating experimental data. TGW was responsible for the study design, coordination, treatment planning and contributed to the final draft. MW advised statistical procedures, recalculated results and contributed to the draft. NB carried out MRI fusions and participated in the statistical analysis of the results and helped revising the draft. TW carried out the treatment planning and helped revising the draft. All authors read and approved the final manuscript.

\section{Author details}

'Department of Radiation Oncology, Jena University Hospital, Friedrich-Schiller-University Jena, Bachstrasse 18, Jena D-07743, Germany. ${ }^{2}$ Department of Radiation Oncology, Division of Medical Physics, Jena University Hospital, Friedrich-Schiller-University Jena, Bachstrasse 18, Jena D-07743, Germany. ${ }^{3}$ Institute of Medical Statistics, Computer Sciences and
Documentation, Jena University Hospital, Friedrich-Schiller-University Jena, Bachstrasse 18, Jena D-07743, Germany.

Received: 1 April 2013 Accepted: 21 July 2013

Published: 24 July 2013

\section{References}

1. Kempermann G: The neurogenic reserve hypothesis: what is adult hippocampal neurogenesis good for? Trends Neurosci 2008, 31:163-169.

2. Doetsch F, Caillé I, Lim DA, García-Verdugo JM, Alvarez-Buylla A: Subventricular zone astrocytes are neural stem cells in the adult mammalian brain. Cell 1999, 97:703-716.

3. Gage FH: Mammalian neural stem cells. Science 2000, 287(5457):1433-1438.

4. Mizumatsu S, Monje ML, Morhardt DR, Rola R, Palmer TD, Fike JR: Extreme sensitivity of adult neurogenesis to low doses of X-irradiation. Cancer Res 2003, 63:4021-4027.

5. Correa DD: Neurocognitive Function in Brain Tumors. Current Neurol Neuroscience Rep 2010, 10:232-239.

6. Dietrich J, Monje M, Wefel J, Meyers C: Clinical patterns and biological correlates of cognitive dysfunction associated with cancer therapy. Oncologist 2008, 13:1285-1295.

7. Chera BS, Amdur RJ, Patel P, Mendenhall WM: A Radiation Oncologist's Guide to Contouring the Hippocampus. Am J Clin Oncol-Cancer Clinical Trials 2009, 32:20-22.

8. Barani IJ, Cuttino LW, Benedict SH, Todor D, Bump E, Wu Y, Chung T, Broaddus W, Lin P: Neural stem cell-preserving external beam radiotherapy of central nervous system malignancies. Int J Radiat Oncol Biol Phys 2007, 68:978-985.

9. Feuvret L, NoËl G, Mazeron J-J, Bey P: Conformity Index: A review. Int J Radiat Oncol Biol Phys 2006, 64:333-342.

10. Kappadakunnel M, Eskin A, Dong J, Nelson SF, Mischel PS, Liau LM, Ngheimphu P, Lai A, Cloughesy TF, Goldin J, Pope WB: Stem cell associated gene expression in glioblastoma multiforme: relationship to survival and the subventricular zone. J Neurooncol 2010, 96:359-367.

11. Lim DA, Cha S, Mayo MC, Chen MH, Keles E, Vandenberg S, Berger MS: Relationship of glioblastoma multiforme to neural stem cell regions predicts invasive and multifocal tumor phenotype. Neuro Oncol 2007, 9:424-428.

12. Bland JM, Altman DG: Statistical methods for assessing agreement between two methods of clinical measurement. Lancet 1986, 8476:307-310.

13. Kortmann RD, Timmermann B, Taylor RE, Scarzello G, Plasswilm L, Paulsen F, Jeremic B, Gnekow AK, Dieckmann K, Kay S, Bamberg M: Current and Future Strategies in Radiotherapy of Childhood Low-Grade Glioma of the Brain. Part II: Treatment-Related Late Toxicity. Strahlenther Onkol 2003, 179:585-597.

14. Balducci M, Apicella G, Manfrida S, Mangiola A, Fiorentino A, Azario L, D'Agostino GR, Frascino V, Dinapoli N, Mantini G, Albanese A, De Bonis P, Chiesa S, Valentini V, Anile C, Cellini N: Single-arm phase II study of conformal radiation therapy and temozolomide plus fractionated stereotactic conformal boost in high-grade gliomas: final report. Strahlenther Onkol 2010, 186:558-564.

15. Baumert BG, Lutterbach J, Bernays R, Davis JB, Heppner FL: Fractionated stereotactic radiotherapy boost after post-operative radiotherapy in patients with high-grade gliomas. Radiother Oncol 2003, 67:183-190.

16. Fokas E, Wacker U, Gross MW, Henzel M, Encheva E, Engenhart-Cabillic R, 185: Hypofractionated stereotactic reirradiation of recurrent glioblastomas. A beneficial treatment option after high-dose radiotherapy? Strahlenther Onkol 2009, 185:235-240.

17. Henke G, Paulsen F, Steinbach JP, Ganswindt U, Isijanov H, Kortmann RD, Bamberg M, Belka C: Hypofractionated reirradiation for recurrent malignant glioma. Strahlenther Onkol 2009, 185:113-119.

18. Chang EL, Wefel JS, Hess KR, Allen PK, Lang FF, Kornguth DG, Arbuckle RB, Swint JM, Shiu AS, Maor MH, Meyers CA: Neurorecognition in patients with brain metastases treated with radiosurgery or radiosurgery plus wholebrain irradiation: a randomised controlled trial. Lancet Oncol 2009, 10:1037-1044

19. Laack NN, Brown PD, Ivnik RJ, Furth AF, Ballman KV, Hammack JE, Arusel RM, Shaw EG, Buckner JC: Cognitive function after radiotherapy for supratentorial low grade glioma: a North Central Cancer Treatment Group prospective study. Int I Radiat Oncol Biol Phys 2005, 63:1175-1183.

20. Van den Bent MJ, Brandes AA, Taphoorn MJ, Kros JM, Kouwenhoven MC, Delattre JY, Bernsen HJ, Frenay M, Tijssen CC, Grisold W, Sipos L, Enting RH, French PJ, Dinjens WN, Vecht CJ, Allgeier A, Lacombe D, Gorlia T, Hoang- 
Xuan K: Adjuvant procarbazine, lomustine, and vincristine chemotherapy in newly diagnosed anaplastic oligodendroglioma: long-term follow-up of EORTC brain tumor group study 26951. J Clin Oncol 2013, 31:344-350.

21. Welzel G, Fleckenstein K, Mai SK, Hermann B, Kraus-Tiefenbacher U, Wenz F: Acute Neurocognitive Impairment during Cranial Radiation Therapy in Patients with Intracranial Tumors. Strahlenther Onkol 2008, 184:647-654.

22. Abayomi OK: Pathogenesis of Cognitive Decline Following Therapeutic Irradiation for Head and Neck Tumors. Acta Oncol 2002, 41:346-351.

23. Monje ML, Vogel H, Masek M, Ligon KL, Fisher PG, Palmer TD: Impaired human hippocampal neurogenesis after treatment for central nervous system malignancies. Ann Neurol 2007, 62:515-520.

24. Kempermann $\mathrm{G}$ : Seven principles in the regulation of adult neurogenesis. Eur J Neurosci 2011, 33:1018-1024.

25. Shors TJ, Townsend DA, Zhao M, Kozorovitskiy Y, Gould E: Neurogenesis may relate to some but not all types of hippocampal-dependent learning. Hippocampus 2002, 12:578-584.

26. Saxe MD, Battaglia F, Wang JW, Malleret G, David DJ, Monckton JE, Garcia AD, Sofroniew MV, Kandel ER, Santarelli L, Hen R, Drew MR: Ablation of hippocampal neurogenesis impairs contextual fear conditioning and synaptic plasticity in the dentate gyrus. Proc Natl Acad Sci U S A 2006, 103:17501-17506.

27. Fike JR, Rosi S, Limoli CL: Neural Precursor Cells and CNS Radiation Sensitivity. Semin Radiat Oncol 2009, 19:22-32.

28. Lazarini F, Mouthon M-A, Gheusi G, De Chaumont F, Olivo-Marin J-C, Lamarque S, Abrous DN, Boussin FD, Lledo PM: Cellular and Behavioral Effects of Cranial Irradiation of the Subventricular Zone in Adult Mice. PLoS One 2009, 4:e7017.

29. Peißner W, Kocher $M$, Treuer $H$, Gillardon F: lonizing radiation-induced apoptosis of proliferating stem cells in the dentate gyrus of the adult rat hippocampus. Molecul Brain Res 1999, 71:61-68.

30. Rola R, Fishman K, Baure J, Rosi S, Lamborn KR, Obenaus A, Nelson GA, Fike JR: Hippocampal Neurogenesis and Neuroinflammation after Cranial Irradiation with 56Fe Particles. Radiat Res 2008, 169:626-632.

31. De La Fuente Herman T, Ahmada S, Vlachaki MT: Intensity modulated radiation therapy versus three dimensional conformal radiation therapy for treatment of high grade glioma: A radiobiological modeling study. J X-ray Science Technol 2010, 18:393-402.

32. Gondi V, Tolakanahalli R, Mehta MP, Tewatia D, Rowley H, Kuo JS, Khuntia D, Tomé WA: Hippocampal-Sparing Whole-Brain Radiotherapy: A "How-To" Technique Using Helical Tomotherapy and Linear Accelerator-Based Intensity-Modulated Radiotherapy. Int J Radiat Oncol Biol Phys 2010, 78:1244-1252

33. Marsh JC, Gielda BT, Herskovic AM, Wendt JA, Turian JV: Sparing of the hippocampus and limbic circuit during whole brain radiation therapy: A dosimetric study using helical tomotherapy. J Med Imag Radiat Oncol 2010, 54:375-382.

34. Zach L, Stall B, Ning H, Ondos J, Arora B, Uma S, Miller RW, Citrin D, Camphausen $\mathrm{K}$ : A dosimetric comparison of four treatment planning methods for high grade glioma. Radiat Oncol 2009, 4:45

35. Gondi V, Hermann BP, Mehta MP, Tomé WA: Hippocampal Dosimetry Predicts Neurocognitive Function Impairment After Fractionated Stereotactic Radiotherapy for Benign or Low-Grade Adult Brain Tumors. Int J Radiation Oncol Biol Phys 2013, 85(2):348e-354e.

doi:10.1186/1748-717X-8-187

Cite this article as: Oehler et al: Neural stem cell sparing by linac based intensity modulated stereotactic radiotherapy in intracranial tumors. Radiation Oncology 2013 8:187.

\section{Submit your next manuscript to BioMed Central and take full advantage of:}

- Convenient online submission

- Thorough peer review

- No space constraints or color figure charges

- Immediate publication on acceptance

- Inclusion in PubMed, CAS, Scopus and Google Scholar

- Research which is freely available for redistribution

Submit your manuscript at www.biomedcentral.com/submit
C Biomed Central 